\title{
The Economic Burden of Comorbid Obstructive Sleep Apnea Among Patients with Chronic Obstructive Pulmonary Disease
}

\author{
Yoon Duk Hong, PharmD; Eberechukwu Onukwugha, MS, PhD; and Julia F. Slejko, PhD
}

\begin{abstract}
BACKGROUND: Chronic obstructive pulmonary disease (COPD) imposes a substantial burden on patients and the health care system. The presence of comorbid obstructive sleep apnea (OSA) has been shown to increase the risk of morbidity and mortality in patients with COPD. There is limited information available on the incremental economic burden of comorbid OSA among patients with COPD.
\end{abstract}

OBJECTIVE: To estimate the incremental health care resource utilization (HCRU) and direct medical costs associated with having comorbid OSA among individuals with COPD in a nationally representative commercially insured population in the United States.

METHODS: We identified individuals with a diagnosis of COPD between January 2008 and December 2014, with and without OSA, from the IQVIA PharMetrics Plus database. The index date was defined as the first claim with a diagnosis of COPD. All baseline characteristics were measured in the 12-month pre-index period, and all outcomes were measured in the 12-month post-index period. The odds of experiencing one or more hospitalizations and emergency room (ER) visits were compared between individuals with and without comorbid OSA using logistic regression models. Twelve-month total, physician office visit, and other outpatient costs were compared between individuals with and without OSA using generalized linear models. To account for a high proportion of zero costs, 2-part models were fit to examine inpatient, ER visit, and pharmacy costs. Average marginal costs were estimated to compare the costs of individuals with and without OSA. All costs represented direct medical costs from the health plan perspective.

RESULTS: Following application of inclusion and exclusion criteria, the study sample included 85,940 individuals with COPD alone and 7,942 individuals with COPD and OSA. The odds of experiencing a hospitalization and an ER visit were significantly higher in the COPD-OSA cohort than in the COPD-only cohort (hospitalization $\mathrm{OR}=1.45,95 \% \mathrm{Cl}=13531$.38-1.53; ER visit $\mathrm{OR}=1.24,95 \% \mathrm{Cl}=1.18-1.30$ ). The average difference in total cost between individuals with and without comorbid OSA was \$8,144 (95\% $\mathrm{Cl}=\$ 7,295-\$ 8,993)$. The average difference in costs for physician office visits and other outpatient services was $\$ 392(95 \% \mathrm{Cl}=\$ 351-\$ 433)$ and $\$ 2,831(95 \% \mathrm{Cl}=\$ 2,463-\$ 3,200)$, respectively. Among individuals with a non-zero, strictly positive inpatient cost, the average difference in inpatient costs was $\$ 2,792(95 \% \mathrm{Cl}=\$ 1,354-\$ 4,230)$. Similarly, among individuals with strictly positive pharmacy and ER costs, the average difference in costs between individuals with and without comorbid OSA was $\$ 1,772$ $(95 \% \mathrm{Cl}=\$ 1,590-\$ 1,953)$ and $\$ 144(95 \% \mathrm{Cl}=\$ 101-\$ 188)$, respectively.

CONCLUSIONS: Total medical cost and costs for inpatient, ER, pharmacy, physician office visit, and other outpatient services were higher among COPD patients with comorbid OSA compared to patients without. The economic burden of comorbid OSA among patients with COPD in the commercially insured U.S. population is substantial.

J Manag Care Spec Pharm. 2020;26(10):1353-62

Copyright $\odot 2020$, Academy of Managed Care Pharmacy. All rights reserved.

\section{What is already known about this subject}

Studies have shown that comorbid obstructive sleep apnea (OSA) increases the risk of exacerbations and mortality in patients with chronic obstructive pulmonary disease (COPD).

Comorbid sleep apnea syndrome was documented to be associated with higher medical utilization and costs in COPD patients in the Maryland Medicaid population.

\section{What this study adds}

To our knowledge, this is the first study to examine the economic burden of comorbid OSA among COPD patients in the commercially insured U.S. population.

Patients diagnosed with COPD and OSA had significantly higher all-cause 12-month health care costs (total, inpatient, emergency room, pharmacy, physician office visit, and other outpatient) than patients without OSA.

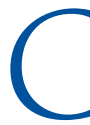
hronic obstructive pulmonary diseases (COPD) and obstructive sleep apnea (OSA) are highly prevalent respiratory diseases that impose a substantial burden on individuals, the health care system, and society. ${ }^{1,2}$ COPD is a disease that can have a high burden of physical symptoms, such as shortness of breath and fatigue, compromising patients' ability to perform daily living activities. ${ }^{3,4}$ COPD has also been associated with a decline in mental health and health-related quality of life..$^{5}$ In 2011, approximately 14 million adults in the United States reported having a COPD diagnosis, ${ }^{6}$ and COPD was the fourth leading cause of U.S. deaths in 2016. ${ }^{7}$ Additionally, COPD was estimated to have an economic impact of $\$ 49.9$ billion in 2010 due to direct and indirect health care costs. ${ }^{8}$

OSA is estimated to affect 29.4 million U.S. adults, representing $12 \%$ of the adult population. ${ }^{9}$ OSA has also been shown to impose a substantial economic burden, with the estimated cost of diagnosing and treating OSA in the United States being approximately $\$ 12.4$ billion in $2015 .{ }^{9}$

The coexistence of OSA and COPD in individuals has been reported to range from $2.9 \%$ to as high as $65.9 \% .{ }^{10}$ When COPD and OSA coexist in the so-called overlap syndrome, increased risk for morbidity and mortality have been reported. ${ }^{11}$ The presence of OSA has been shown to increase COPD exacerbations and mortality when compared with COPD-only patients. ${ }^{11}$ Additionally, COPD patients with comorbid OSA have been 
reported to have poorer health-related quality of life, partly due to worsened sleep disturbance and more pronounced nocturnal oxygen desaturation. ${ }^{12}$

While the clinical burden of COPD-OSA overlap syndrome has been studied, the economic burden has not been fully quantified in the United States. In a study conducted using Maryland Medicaid claims data (2001-2003), beneficiaries with COPD and sleep apnea syndrome incurred higher annual medical costs compared with beneficiaries without sleep apnea syndrome, highlighting the financial burden of COPD and comorbid sleep apnea in the Maryland Medicaid population. ${ }^{13}$ However, the incremental economic burden of OSA in commercially insured U.S. COPD patients is yet unknown.

A better understanding of the incremental economic burden of comorbid OSA among COPD patients will help create the context for important decisions such as whether reimbursement mechanisms for OSA diagnostic testing and treatment should be reevaluated to improve patient access to treatment and whether COPD patients should be actively screened and treated for OSA. Accurate and current estimates of health care resource utilization (HCRU) and costs are warranted to better understand the added economic burden of comorbid OSA in patients with COPD, and the economic benefits that may potentially be achieved by optimizing the treatment of patients with COPD and comorbid OSA and lowering the risk of adverse health outcomes.

The objective of this study was to estimate the incremental HCRU and direct medical costs associated with having comorbid OSA among individuals with COPD in a nationally representative U.S. commercially insured population. The analysis was performed from the U.S. commercial payer perspective.

\section{Methods}

\section{Study Design and Data Source}

The 10\% random sample of enrollees within the IQVIA PharMetrics Plus database was used for this retrospective cohort study. The PharMetrics Plus database is composed of fully adjudicated health plan claims data and enrollment information for over 190 million commercially insured individuals since 2006 from national and subnational health plans and self-insured employer groups throughout the United States. ${ }^{14}$ This study was deemed exempt by the Institutional Review Board at the University of Maryland, Baltimore.

\section{Study Sample}

Individuals aged 18 years and older were included in the study if they had at least 1 claim with a COPD diagnosis code (International Classification of Diseases, Ninth Revision, Clinical Modification [ICD-9-CM] codes 491.xx, 492.xx, and 496.xx) between January 1, 2008, and December 31, 2014. The index date was defined as the date of the first claim with a COPD diagnosis during the study time frame. All individuals were required to have continuous enrollment during the 12 months before the index date to capture pre-index baseline characteristics and 12 months after the index date to ensure complete outcome measurement during follow-up. Individuals with COPD and comorbid asthma (ICD-9-CM diagnosis codes 493.0x, 493.1x, 493.2x, 493.8x, and 493.9x), that is, asthmaCOPD overlap syndrome, in the 12-month pre-index period were excluded from the study, since these individuals are known to be clinically different from individuals with COPD only and incur higher health care costs. ${ }^{15}$

\section{Study Cohorts}

Individuals with COPD were split into 2 mutually exclusive cohorts on the basis of their OSA status in the pre-index period, which was our main exposure of interest. Individuals with at least 1 claim including a diagnosis of OSA (ICD-9-CM diagnosis code 327.23) during the 12-month pre-index period were categorized as individuals with COPD and comorbid OSA; individuals without a diagnosis of OSA were categorized as individuals with COPD without OSA.

\section{Baseline Measures}

Baseline characteristics including age, sex, and geographic region were measured at the index date. Comorbidity burden was measured using the Charlson Comorbidity Index with Quan modification in the 12-month pre-index period. ${ }^{16}$ Because obesity has been shown to be correlated with OSA and higher health care cost, the presence of a diagnosis of obesity (ICD-9-CM diagnosis code 278.xx) was identified in the 12-month pre-index period. Pre-index oxygen use and COPD-related hospitalizations were used as proxy measures for COPD severity. ${ }^{17}$

\section{Outcome Measures}

We examined the number of hospitalizations and ER visits per patient in the 12-month post-index period, and the odds of experiencing 1 or more hospitalizations and ER visits were compared between the 2 cohorts. We calculated all-cause total medical cost per patient in the 12-month post-index period by summing all costs from the claims files over that period. We also stratified the cost by HCRU category (inpatient, ER, pharmacy, physician office visit, and other outpatient, which included lab/pathology, radiology, and outpatient surgical or rehabilitation services). Costs were compared between individuals with and without OSA as a ratio and as the average marginal cost. All costs evaluated were direct medical costs obtained from the dollar amount paid by the health plan to a provider/facility for services rendered. Costs were adjusted to 2018 U.S. dollars using the annual medical care component of the Consumer Price Index to account for inflation. ${ }^{18}$ 
The Economic Burden of Comorbid Obstructive Sleep Apnea

Among Patients with Chronic Obstructive Pulmonary Disease

\begin{tabular}{|c|c|c|c|c|c|}
\hline \multirow[b]{2}{*}{ Age in years, mean (SD) } & \multicolumn{2}{|c|}{ Individuals with COPD $(n=85,940)$} & \multicolumn{2}{|c|}{$\begin{array}{l}\text { Individuals with COPD and OSA } \\
\qquad(\mathrm{n}=7,942)\end{array}$} & \multirow{2}{*}{$\frac{\text { P Value }^{\mathrm{a}}}{<0.01}$} \\
\hline & 60.7 & $(11.9)$ & 59.3 & $(10.5)$ & \\
\hline \multicolumn{5}{|l|}{ Age in years, $n(\%)$} & $<0.01$ \\
\hline$<40$ & 3,517 & $(4.09)$ & 264 & $(3.32)$ & \\
\hline $40-54$ & 21,442 & $(24.95)$ & 2,153 & $(27.11)$ & \\
\hline $55-64$ & 31,295 & $(36.41)$ & 3,296 & $(41.50)$ & \\
\hline$\geq 65$ & 29,686 & $(34.54)$ & 2,229 & $(28.07)$ & \\
\hline \multicolumn{5}{|l|}{ Sex, n (\%) } & $<0.01$ \\
\hline Female & 43,358 & $(50.45)$ & 3,105 & $(39.10)$ & \\
\hline Male & 42,567 & $(49.53)$ & 4,836 & $(60.89)$ & \\
\hline \multicolumn{5}{|l|}{ Geographic region, $\mathrm{n}(\%)$} & $<0.01$ \\
\hline East & 22,395 & $(26.06)$ & 1,836 & $(23.12)$ & \\
\hline South & 28,774 & $(33.48)$ & 2,779 & $(34.99)$ & \\
\hline Midwest & 25,489 & $(29.66)$ & 2,505 & $(31.54)$ & \\
\hline West & 9,282 & $(10.80)$ & 822 & $(10.35)$ & \\
\hline \multicolumn{5}{|c|}{ Pre-index oxygen use, n (\%) } & $<0.01$ \\
\hline No & 80,646 & $(93.84)$ & 6,496 & $(81.79)$ & \\
\hline Yes & 5,294 & $(6.16)$ & 1,446 & $(18.21)$ & \\
\hline \multicolumn{5}{|c|}{ Pre-index COPD-related hospitalization, n (\%) } & $<0.01$ \\
\hline No & 81,916 & $(95.32)$ & 7,459 & $(93.92)$ & \\
\hline Yes & 4,024 & $(4.68)$ & 483 & $(6.08)$ & \\
\hline \multicolumn{5}{|l|}{ CCI, n (\%) } & $<0.01$ \\
\hline 1 & 65,919 & $(76.70)$ & 5,377 & $(67.70)$ & \\
\hline$\geq 2$ & 20,021 & $(23.30)$ & 2,565 & $(32.30)$ & \\
\hline
\end{tabular}

\section{Statistical Analysis}

Chi-square tests and Student's t-tests were performed to compare baseline characteristics, unadjusted rates of hospitalizations and ER visits experienced, and costs of the COPDonly and COPD-OSA cohorts. Chi-square tests were used to compare categorical data, and Student's t-tests were used to compare continuous data.

A logistic regression was performed to compare the odds of experiencing 1 or more hospitalizations and ER visits in the COPD-OSA cohort versus the COPD-only cohort. A generalized linear model (GLM) with a gamma distribution and a log link was fit to compare total costs, physician office visit costs, and other outpatient costs for individuals with comorbid OSA versus individuals without OSA. To account for a high proportion of zero costs, a two-part model was fit to examine inpatient, ER visit, and pharmacy costs. The first part of the model consisted of a logit model estimating the odds of having any costs, and the second part was a GLM with a gamma distribution and a log link modeling the expected cost conditional on having non-zero, strictly positive costs.

The average marginal total cost and the average marginal cost for each HCRU category were estimated by computing the marginal effect at each observation level and then calculating the sample average of individual marginal effects to obtain the overall average marginal effect. ${ }^{19}$ The $95 \%$ confidence intervals (CIs) for the average marginal costs were estimated using the delta method. Baseline characteristics were adjusted for in all analyses. We were unable to report or include obesity as a covariate in the analyses because few individuals had evidence of this diagnosis. A modified Parks test was used to determine data support for the assumption that the cost and HCRU data follow the distribution chosen for the GLM, ${ }^{20}$ and Ramsey's Regression Equation Specification Error Test (RESET) was performed to verify the link. ${ }^{21}$ All analyses were performed using SAS Studio (SAS Institute, Cary, NC), and a $P$ value less than 0.05 was considered statistically significant.

\section{Sensitivity Analysis}

To test the robustness of the estimated outcomes, a sensitivity analysis was conducted for all outcomes with additional covariates for index year (2008, 2009, 2010, 2011, 2012, 2013, and 2014) added to the model. This was done to ensure that changes in clinical practice patterns and new COPD treatments approved within the study time period had no effect on the estimated outcomes. We also conducted a sensitivity analysis excluding individuals with a diagnosis code for obesity to examine if this led to changes in the overall results. 


\begin{tabular}{|c|c|c|c|c|c|}
\hline & \multicolumn{2}{|c|}{$\begin{array}{c}\text { Individuals with COPD } \\
\qquad(\mathrm{n}=85,915)\end{array}$} & \multicolumn{2}{|c|}{$\begin{array}{l}\text { Individuals with COPD and OSA } \\
\qquad(\mathrm{n}=7,942)\end{array}$} & \multirow[b]{2}{*}{$P$ Value } \\
\hline & n & $\%$ & n & $\%$ & \\
\hline No hospitalizations & 59,429 & 69.2 & 4,705 & 59.2 & $<0.01$ \\
\hline$\geq 1$ hospitalization & 26,486 & 30.8 & 3,237 & 40.8 & \\
\hline No ER visits & 57,691 & 67.2 & 4,882 & 61.5 & $<0.01$ \\
\hline \multirow[t]{2}{*}{$\geq 1$ ER visit } & 28,224 & 32.9 & 3,060 & 38.5 & \\
\hline & Mean & SD & Mean & SD & $P$ Value \\
\hline Number of hospitalizations (including nonutilizers) & 0.55 & 1.20 & 0.77 & 1.43 & $<0.01$ \\
\hline Number of hospitalizations if $\geq 1$ hospitalization & 1.80 & 1.55 & 1.90 & 1.69 & $<0.01$ \\
\hline Number of ER visits (including nonutilizers) & 1.76 & 6.19 & 2.12 & 8.62 & $<0.01$ \\
\hline Number of ER visits if $\geq 1$ ER visit & 5.36 & 9.87 & 5.49 & 13.20 & 0.49 \\
\hline
\end{tabular}

Additionally, we changed the inclusion criteria to require at least 2 claims with a COPD diagnosis code, at least 30 days apart, to examine if this led to changes in the estimated outcomes.

\section{Results}

\section{Baseline Characteristics}

A total of 93,882 patients with COPD met the study inclusion and exclusion criteria. A flowchart showing the selection of the study sample is available in Appendix A (available in online article), and patients' baseline characteristics are shown in Table 1. Twenty-five individuals only had claims that were denied, so those individuals were excluded from the analysis. We included 85,915 patients with COPD without OSA and 7,942 patients with COPD and OSA in the analysis.

\section{HCRU: Hospitalizations/ER Visits-Descriptive Analysis}

Unadjusted rates of hospitalizations and ER visits are presented in Table 2. A higher proportion of individuals with comorbid OSA experienced hospitalizations and ER visits compared with individuals without OSA (40.8\% vs. $30.8 \%$; $P<0.01$ for hospitalizations; $38.5 \%$ vs. $32.9 \%$; $P<0.01$ for ER visits). The mean number of hospitalizations and ER visits were both higher in the COPD-OSA cohort ( 0.77 vs. $0.55 ; P<0.01$ for hospitalizations; 2.12 vs. $1.76 ; P<0.01$ for ER visits).

\section{HCRU: Hospitalizations/ER Visits - Adjusted Regression Analysis}

Adjusted for baseline characteristics, the odds of experiencing a hospitalization were significantly higher in the COPD-OSA cohort than in the COPD-only cohort (odds ratio $[\mathrm{OR}]=1.45$; 95\% CI $=1.38-1.53 ; P<0.01)$. The odds of experiencing an ER visit were also significantly higher in the COPD-OSA cohort $(\mathrm{OR}=1.24 ; 95 \% \mathrm{CI}=1.18-1.30 ; \mathrm{P}<0.01)$.

\section{Health Care Costs-Descriptive Analysis}

The unadjusted mean 12-month costs of individuals in the COPD-OSA cohort and the COPD-only cohort are presented in Table 3. The mean (SD) 12-month total cost for individuals with COPD and comorbid OSA was $\$ 32,566(\$ 61,981)$ compared with $\$ 20,342(\$ 47,472)$ for individuals with COPD without OSA. Individuals with comorbid OSA also had higher inpatient, ER, pharmacy, physician office visit, and other outpatient costs than individuals with COPD only $(P<0.01)$. Inpatient and other outpatient costs represented the majority of costs in both cohorts.

\section{Health Care Costs-Adjusted Regression Analysis}

Total costs of individuals with COPD and comorbid OSA were estimated to be $40 \%$ higher than individuals with COPD only $(P<0.01$; Table 4). Physician office visit and other outpatient costs were also estimated to be $45 \%$ and $37 \%$ higher for individuals with COPD and comorbid OSA, respectively $(P<0.01)$. The odds of having any inpatient, ER, and pharmacy costs among those with COPD and comorbid OSA were 45\%, 27\%, and $22 \%$ higher, respectively, than individuals with COPD only $(P<0.01)$. Among individuals with non-zero, strictly positive inpatient, ER, and pharmacy costs, inpatient costs were estimated to be $10 \%$ higher for individuals with comorbid OSA compared with individuals without $(P<0.01)$; ER costs were estimated to be $17 \%$ higher for individuals with comorbid OSA compared with individuals without $(P<0.01)$; and pharmacy costs were estimated to be $46 \%$ higher for individuals with OSA $(P<0.01$; Table 4$)$.

The average marginal total cost (i.e., the average difference in total cost between individuals with and without comorbid OSA) was $\$ 8,144$ (95\% CI $=\$ 7,295-\$ 8,993 ; P<0.01$; Table 5). The average marginal cost for other outpatient services was $\$ 2,831(95 \% C I=\$ 2,463-\$ 3,200 ; P<0.01)$, and the 
The Economic Burden of Comorbid Obstructive Sleep Apnea

Among Patients with Chronic Obstructive Pulmonary Disease

TABLE 3 Unadjusted Mean 12-Month Costs in the COPD-Only Cohort and the COPD-OSA Cohort

\begin{tabular}{|c|c|c|c|c|c|c|}
\hline \multirow[b]{3}{*}{ Total cost } & \multicolumn{2}{|c|}{ Individuals with COPD $(n=85,915)$} & \multicolumn{3}{|c|}{ Individuals with COPD and OSA $(\mathrm{n}=7,942)$} & \multirow{3}{*}{$\frac{P \text { Value }}{<0.01}$} \\
\hline & Mean (SD) & \multirow{2}{*}{$\begin{array}{c}\% \\
100.0\end{array}$} & \multicolumn{2}{|c|}{ Mean (SD) } & \multirow{2}{*}{$\begin{array}{c}\% \\
100.0\end{array}$} & \\
\hline & $\$ 20,342 \quad(\$ 47,472)$ & & $\$ 32,566$ & $(\$ 61,981)$ & & \\
\hline \multicolumn{7}{|c|}{ Cost per HCRU category } \\
\hline Inpatient & $\$ 8,512 \quad(\$ 34,535)$ & 41.8 & $\$ 14,041$ & $(\$ 46,029)$ & 43.1 & $<0.01$ \\
\hline ER visit & $(\$ 1,163)$ & 1.3 & $\$ 397$ & $(\$ 1,510)$ & 1.2 & $<0.01$ \\
\hline Pharmacy & $(\$ 7,638)$ & 14.8 & $\$ 5,011$ & $(\$ 13,233)$ & 15.4 & $<0.01$ \\
\hline Physician office visit & $(\$ 1,104)$ & 4.3 & $\$ 1,282$ & $(\$ 1,476)$ & 3.9 & $<0.01$ \\
\hline Other outpatient & $\$ 7,689 \quad(\$ 21,575)$ & 37.8 & $\$ 11,837$ & $(\$ 25,308)$ & 36.3 & $<0.01$ \\
\hline
\end{tabular}

average marginal cost for physician office visits was $\$ 392$ (95\% $\mathrm{CI}=\$ 351-\$ 433 ; \mathrm{P}<0.01$ ). The average marginal probability of having a non-zero, strictly positive inpatient, ER visit, and pharmacy cost from the logit part of the 2-part models are presented in Appendix B (available in online article). Among individuals with a non-zero, strictly positive inpatient cost, the average marginal cost for inpatient stays was $\$ 2,792$ (95\% $\mathrm{CI}=\$ 1,354-\$ 4,230 ; \quad P<0.01)$. Similarly, among individuals with a non-zero, strictly positive pharmacy cost, the average marginal cost for pharmacy services was $\$ 1,772$ (95\% $\mathrm{CI}=\$ 1,590-\$ 1,953 ; P<0.01)$. The average marginal cost for ER visits, given that individuals have a non-zero, strictly positive ER cost, was $\$ 144$ (95\% CI $=\$ 101-188 ; P<0.01)$.

\section{Sensitivity Analysis}

The results from the sensitivity analysis remained consistent with the original analysis. Adjusting for index year did not have a significant effect on the outcome estimates. The odds of experiencing a hospitalization in the COPD-OSA cohort was 1.43 times the odds of experiencing a hospitalization in the COPD-only cohort (OR=1.43; 95\% CI=1.36-1.51). The odds of experiencing an ER visit was also higher in the COPD-OSA cohort $(\mathrm{OR}=1.22 ; 95 \% \mathrm{CI}=1.16-1.28)$. Total cost was estimated to be 1.38 times higher in the COPD-OSA cohort $(P<0.01)$. Office visit costs were found to be 1.45 times higher in the COPD-OSA cohort $(P<0.01)$, and other outpatient costs were found to be 1.35 times higher $(P<0.01)$. The relative cost ratios for inpatient, ER visit, and pharmacy costs remained consistent with the results of the original analysis.

The average marginal total cost of $\$ 7,731$ ( $95 \% \mathrm{CI}=\$ 6,891$ $\$ 8,571$ ), and the average marginal cost for each HCRU category were similar to the estimates from the original analysis. Additionally, excluding individuals with a diagnosis of obesity did not change the results of the adjusted regression analyses. Requiring at least 2 claims with a COPD diagnosis to be included in the study sample also yielded outcome estimates that were similar to the estimates from the original analysis.

\section{Discussion}

Knowledge about the economic burden of illnesses is useful when examining unmet need, evaluating the appropriateness of current policies, and planning and implementing new health policy interventions. It is well known that COPD is associated with high health care costs. ${ }^{22-24}$ Similar to what was observed in this study, high annual health care costs have been reported in the literature. In an analysis of commercially insured individuals with COPD in the United States, ${ }^{22}$ annual direct costs were estimated to range between $\$ 11,395$ and $\$ 17,651$ (2010 U.S. dollars) based on exacerbation frequency. The estimated costs are lower than the costs observed in our study, which is most likely because the study only included working-age individuals aged 65 years and younger. ${ }^{22}$ In another study of commercially insured patients with COPD, the mean annual health care cost was estimated to range from $\$ 2,003$ to as high as $\$ 43,461$ (2008 U.S. dollars), depending on the level of care received..$^{25}$

It is important to recognize that patients with COPD often have comorbidities, which may independently contribute to the economic burden of illness in the COPD population. While the clinical outcomes of COPD patients with comorbid OSA have been studied, ${ }^{11,12,26}$ the economic burden associated with OSA among COPD patients in the commercially insured U.S. population has not been assessed. This study offers an assessment of the health care cost burden associated with comorbid OSA among commercially insured COPD patients in the United States. We used a large sample of individuals represented in an administrative claims database. The database includes fully adjudicated medical and pharmacy claims and thus presents an ideal data source for studying the cost of covered health services among individuals diagnosed with COPD only and with comorbid OSA. The results from this study may prompt and guide payers and policymakers to design targeted disease management programs for COPD patients with comorbid OSA. 
The Economic Burden of Comorbid Obstructive Sleep Apnea

Among Patients with Chronic Obstructive Pulmonary Disease

TABLE 4 12-Month Cost Regression Results: COPD-OSA Cohort vs. COPD-Only Cohort (Reference)

\begin{tabular}{|c|c|c|c|c|}
\hline & \multicolumn{3}{|c|}{ Cost Ratio $(95 \%$ CI $)$} & $P$ Value \\
\hline Total $\cos ^{\mathrm{a}}$ & \multicolumn{3}{|c|}{$1.40 \quad(1.36-1.44)$} & $<0.01$ \\
\hline \multicolumn{5}{|l|}{ Cost per HCRU category } \\
\hline \multicolumn{5}{|l|}{ One-part models ${ }^{\mathrm{a}}$} \\
\hline Physician office visit & \multicolumn{3}{|c|}{$1.45 \quad(1.40-1.50)$} & $<0.01$ \\
\hline Other outpatient & \multicolumn{3}{|c|}{$\begin{array}{lll} & 1.37 & (1.32-1.42) \\
\end{array}$} & $<0.01$ \\
\hline Two-part models ${ }^{\mathrm{b}}$ & Logit $\mathrm{M}$ & lel Odds Ratio & GLM Cost Ratio & \\
\hline Inpatient & 1.45 & $(1.38-1.53)$ & $1.10 \quad(1.05-1.15)$ & $<0.01^{\mathrm{c}} ;<0.01^{\mathrm{d}}$ \\
\hline ER visit & 1.27 & $(1.21-1.33)$ & $1.17 \quad(1.12-1.22)$ & $<0.01^{\mathrm{c}} ;<0.01^{\mathrm{d}}$ \\
\hline Pharmacy & 1.22 & $(1.15-1.30)$ & $1.46 \quad(1.41-1.51)$ & $<0.01^{\mathrm{c}} ;<0.01^{\mathrm{d}}$ \\
\hline \multicolumn{5}{|c|}{$\begin{array}{l}\text { aGLM with a gamma distribution and a log link was fit. } \\
{ }^{\circ} \text { A 2-part model was fit: (1) GLM (logit link and binomial distribution) and (2) GLM (gamma distribution with a log link). } \\
\text { cP value from logit model. } \\
\text { dP value from GLM. }\end{array}$} \\
\hline
\end{tabular}

Studies have found that patients with COPD and comorbid OSA have worse clinical outcomes and higher HCRU than individuals with COPD alone, including more COPD exacerbations leading to hospitalization, ${ }^{11,27}$ as well as increased risk of cardiovascular events and mortality. ${ }^{11,26}$ In a study examining the effect of comorbidities in COPD patients on HCRU and costs in a predominantly Medicare population, sleep apnea was associated with an $18 \%$ higher all-cause health care costs. ${ }^{27}$ A study of Maryland Medicaid beneficiaries found that individuals with sleep apnea syndrome had higher medical service use and medical cost than beneficiaries without sleep apnea syndrome, with $\$ 4,155$ in excess annual costs. ${ }^{13}$

Similarly, our study comparing HCRU and costs of individuals with COPD living with and without comorbid OSA in a commercially insured population identified significantly increased economic burden attributable to comorbid OSA. The probability of experiencing hospitalizations and ER visits was higher for individuals with comorbid OSA, and these individuals incurred higher health care costs. On average, among those with a non-zero, strictly positive hospitalization cost, having comorbid OSA was associated with approximately $\$ 2,800$ higher costs due to hospitalizations. Additionally, individuals with comorbid OSA incurred higher costs for physician office visits and other outpatient services, which may be due to individuals with OSA presenting with more symptoms or complications, requiring additional work-up and/or treatment.

The results of this study and previous studies suggest that patients with COPD and comorbid OSA are at risk of increased morbidity and are likely to incur higher HCRU and health care costs. It is important to understand the totality of disease burden for patients, not just the single disease state on its own, to align health care services more closely with patients' needs and thus reduce the overall burden of disease. Evidence of the management of comorbid conditions is often limited as patients with comorbid chronic conditions are frequently excluded from randomized clinical trials. ${ }^{28}$ Despite this challenge, clinical practice guidelines should include consideration of comorbidities and how patients should be managed in the presence of these comorbidities.

It was beyond the scope of this study to evaluate whether patients with COPD and comorbid OSA are adequately treated for their OSA. Among COPD patients, an important adverse health event is a COPD exacerbation that may lead to an ER visit and/or hospitalization. Future studies are needed to examine whether treatment for OSA (e.g., positive airway pressure therapy and adherence to such therapy) in this patient population lowers the risk of adverse health events, such as exacerbations leading to hospitalizations, and associated health care costs. Also, it would be interesting to examine whether health care costs differ by OSA severity in this population. It is clear that OSA imposes an added burden on patients with COPD, including an increased risk of experiencing hospitalizations. Optimizing coverage policies and enabling timely patient access to diagnostic testing and treatment and, thus, optimizing the management of this patient population may help reduce this burden. Payers and policymakers may use information from this study to develop and financially plan for tailored therapeutic interventions for improved management of patients with COPD and comorbid OSA.

\section{Limitations}

This study has some limitations to consider. This study was conducted using commercial claims data; therefore, the results cannot be generalized to the U.S. population. Obesity may have been an unmeasured confounder in this study. Except for 


\begin{tabular}{|c|c|c|}
\hline TABLE 5 & $\begin{array}{l}\text { nth Average Margi } \\
\text { OSA Cohort vs. CC } \\
\text { (Reference) }\end{array}$ & $\begin{array}{l}\text { osts: } \\
\text { Only }\end{array}$ \\
\hline & $\begin{array}{l}\text { Average Marginal Cost } \\
\qquad(95 \% \mathrm{CI})^{\mathrm{c}}\end{array}$ & $P$ Value \\
\hline Total cost $\mathrm{t}^{\mathrm{a}}$ & $\$ 8,144 \quad(\$ 7,295-\$ 8,993)$ & $<0.01$ \\
\hline \multicolumn{3}{|l|}{ Cost per HCRU category } \\
\hline Inpatient $\mathrm{t}^{\mathrm{b}}$ & $(\$ 1,354-\$ 4,230)$ & $<0.01$ \\
\hline ER visit ${ }^{b}$ & $(\$ 101-\$ 188)$ & $<0.01$ \\
\hline Pharmacy ${ }^{b}$ & $\$ 1,772 \quad(\$ 1,590-\$ 1,953)$ & $<0.01$ \\
\hline Physician office visit ${ }^{\mathrm{a}}$ & $\$ 392 \quad(\$ 351-\$ 433)$ & $<0.01$ \\
\hline Other outpatient ${ }^{\mathrm{a}}$ & $\$ 2,831 \quad(\$ 2,463-\$ 3,200)$ & $<0.01$ \\
\hline \multicolumn{3}{|c|}{$\begin{array}{l}\text { aGLM with a gamma distribution and a log link was fit. } \\
\text { bSecond part of a 2-part model: GLM (gamma distribution with a log link). Cost } \\
\text { represents the cost given a non-zero cost. } \\
\text { 'Confidence interval estimated using the delta method. } \\
C I=\text { confidence interval; COPD = chronic obstructive pulmonary disease; } \\
\text { ER=emergency room; GLM = generalized linear model; HCRU = health care } \\
\text { resource utilization; OSA = obstructive sleep apnea. }\end{array}$} \\
\hline
\end{tabular}

in the sensitivity analysis, we did not examine the effects of obesity due to a low prevalence, based on the billing code for obesity. Obesity is rarely coded and captured in administrative claims data, and it has been shown that a claims-based diagnosis of obesity lacks sensitivity, leading to an underestimation of the true prevalence. ${ }^{29}$ Additionally, it is possible that the COPD-only cohort included individuals who had OSA but had not been diagnosed, leading to misclassification bias and an underestimation of the true difference.

Despite these limitations, this study provides useful information regarding patterns of health care resource utilization and associated costs. The findings are timely, given the growing evidence regarding multimorbidity and the associated implications for HCRU and costs. ${ }^{30-33}$ To our knowledge, this study is the first to quantify the economic impact of comorbid OSA among commercially insured individuals with COPD from the commercial payer perspective. This study showed that the economic burden of comorbid OSA among individuals with COPD is substantial.

\section{Conclusions}

Individuals with comorbid OSA demonstrated greater HCRU and costs than individuals with COPD alone, with a higher probability of experiencing hospitalizations and ER visits. Rather than treat COPD as a sole condition on its own, the overall disease profile of patients with COPD needs to be considered, since management of the overall comorbid burden of patients may lead to a reduction in adverse health events that lead to ER visits/hospitalizations and potentially a reduction in health care costs.

\section{Authors}

YOON DUK HONG, PharmD; EBERECHUKWU ONUKWUGHA, MS, PhD; and JULIA F. SLEJKO, PhD, Department of Pharmaceutical Health Services Research, University of Maryland School of Pharmacy, Baltimore, Maryland.

AUTHOR CORRESPONDENCE: Yoon Duk Hong, PharmD,

Department of Pharmaceutical Health Services Research, University of Maryland School of Pharmacy, 220 Arch St., 12th Fl., Baltimore, MD 21201. Tel.: 410.706.1418;

Email:yhon039@umaryland.edu.

\section{DISCLOSURES}

No outside funding supported this study. Onukwugha reports grants from Bayer Healthcare Pharmaceuticals and Pfizer, unrelated to this work Slejko reports grants from PhRMA, the PhRMA Foundation, Novartis Pharmaceuticals, and Takeda Pharmaceuticals, along with a teaching honorarium from Pfizer, unrelated to this work. Hong has nothing to disclose.

\section{DATA STATEMENT}

The statements, findings, conclusions, views, and opinions contained and expressed in this article are based in part on data obtained under license from IQVIA (Source: IQVIA PharMetrics Plus, January 2006-December 2015. All Rights Reserved). The statements, findings, conclusions, views, and opinions contained and expressed herein are not necessarily those of IQVIA or any of its affiliated or subsidiary entities.

\section{REFERENCES}

1. Shawon MSR, Perret JL, Senaratna CV, Lodge C, Hamilton GS, Dharmage SC. Current evidence on prevalence and clinical outcomes of co-morbid obstructive sleep apnea and chronic obstructive pulmonary disease: a systematic review. Sleep Med Rev. 2017;32:58-68. doi:10.1016/j. smrv.2016.02.007

2. Global Initiative for Chronic Obstructive Lung Disease. Global strategy for the diagnosis, management and prevention of COPD. 2017 report. Available at: https://goldcopd.org/wp-content/uploads/2017/02/wms-GOLD2017-FINAL.pdf. Accessed September 10, 2020

3. Kessler R, Partridge MR, Miravitlles M, et al. Symptom variability in patients with severe COPD: a pan-European cross-sectional study. Eur Respir J. 2011;37(2):264-72. doi:10.1183/09031936.00051110

4. O'Hagan P, Chavannes NH. The impact of morning symptoms on daily activities in chronic obstructive pulmonary disease. Curr Med Res Opin. 2014;30(2):301-14. doi:10.1185/03007995.2013.857648

5. Miravitlles M, Ribera A. Understanding the impact of symptoms on the burden of COPD. Respir Res. 2017;18(1):67. doi:10.1186/s12931-017-0548-3

6. Ford ES, Croft J, Mannino D, Wheaton A, Zhang X, WH. G. COPD surveillance-United States, 1999-2011. Chest. 2013;144(1):284-305. doi 10.1378/chest.13-0809

7. Heron M. Deaths: leading causes for 2014. Natl Vital Stat Rep. 2016;65(5):1-96. Available at: https://www.cdc.gov/nchs/data/nvsr/nvsr65/ nvsr65_05.pdf. Accessed September 18, 2020.

8. National Heart, Lung, and Blood Institute. Morbidity \& mortality: 2009 chart book on cardiovascular, lung, and blood diseases. 2009. Available at: https://ecopmc.files.wordpress.com/2012/04/2009_chartbook.pdf. Accessed September 10, 2020. 
9. Frost \& Sullivan. Hidden health crisis costing America billions: underdiagnosing and undertreating obstructive sleep apnea draining healthcare system. American Academy of Sleep Medicine. 2016. Available at: https://aasm.org/resources/pdf/sleep-apnea-economic-crisis.pdf. Accessed September 10, 2020.

10. Soler X, Gaio E, Powell FL, et al. High prevalence of obstructive sleep apnea in patients with moderate to severe COPD. Ann Am Thorac Soc. 2015;12(8):1219-25. doi:10.1513/AnnalsATS.201407-336OC

11. Marin JM, Soriano JB, Carrizo SJ, Boldova A, Celli BR. Outcomes in patients with chronic obstructive pulmonary disease and obstructive sleep apnea. Am J Respir Crit Care Med. 2010;182(3):325-31. doi:10.1164/ rccm.200912-18690C

12. Mermigkis C, Kopanakis A, Foldvary-Schaefer N, et al. Health-related quality of life in patients with obstructive sleep apnoea and chronic obstructive pulmonary disease (overlap syndrome). Int J Clin Pract. 2007;61(2):207-11. doi:10.1111/j.1742-1241.2006.01213.x

13. Shaya FT, Lin P-J, Aljawadi MH, Scharf SM. Elevated economic burden in obstructive lung disease patients with concomitant sleep apnea syndrome. Sleep Breath. 2009;13(4):317-23. doi:10.1007/s11325-009-0266-2

14. IQVIA. US Claims - IQVIA PharMetrics Plus. Fact Sheet. May 2020. Available at: https://www.iqvia.com/library/fact-sheets/iqvia-pharmetricsplus. Accessed September 18, 2020.

15. Gerhardsson de Verdier M, Andersson M, Kern DM, Zhou S, Tunceli O. Asthma and chronic obstructive pulmonary disease overlap syndrome: doubled costs compared with patients with asthma alone. Value Health. 2015;18(6):759-66. doi:10.1016/j.jval.2015.04.010

16. Quan H, Li B, Couris CM, et al. Updating and validating the Charlson Comorbidity Index and score for risk adjustment in hospital discharge abstracts using data from 6 countries. Am J Epidemiol. 2011;173(6):676-82. doi:10.1093/aje/kwq433

17. Macaulay D, Sun SX, Sorg RA, et al. Development and validation of a claims-based prediction model for COPD severity. Respir Med. 2013;107(10):1568-77. doi:10.1016/j.rmed.2013.05.012

18. U.S. Bureau of Labor Statistics. Consumer Price Index (CPI) Databases. Available at: https://www.bls.gov/cpi/data.htm. Accessed September 18, 2020.

19. Onukwugha E, Bergtold J, Jain R. A primer on marginal effects—part ii: health services research applications. Pharmacoeconomics. 2015;33(2):97-103. doi:10.1007/s40273-014-0224-0

20. Manning WG, Mullahy J. Estimating log models: to transform or not to transform? J Health Econ. 2001;20(4):461-94. Available at: http://citeseerx. ist.psu.edu/viewdoc/download?doi=10.1.1.494.5616\&rep=repl\&type=pdf. Accessed September 10, 2020

21. Ramsey JB. Tests for specification errors in classical linear least-squares regression analysis. J R Stat Soc Series B Stat Methodol. 1969;31(2):350-71. Available at: https://www.jstor.org/stable/2984219. Accessed September 10, 2020.
22. Patel JG, Coutinho AD, Lunacsek OE, Dalal AA. COPD affects worker productivity and health care costs. Int J COPD. 2018;13:2301-11. doi:10.2147/ COPD.S163795

23. Tinkelman D, Nordyke RJ, Isonaka S, George D, DesFosses K, Nonikov D. The impact of chronic obstructive pulmonary disease on long-term disability costs. J Manag Care Pharm. 2005;11(1):25-32. doi:10.18553/ jmcp.2005.11.1.25

24. Simoni-Wastila L, Blanchette CM, Qian J, et al. Burden of chronic obstructive pulmonary disease in medicare beneficiaries residing in long-term care facilities. Am J Geriatr Pharmacother. 2009;7(5):262-70. doi:10.1016/j.amjopharm.2009.11.003

25. Dalal AA, Christensen L, Liu F, Riedel AA. Direct costs of chronic obstructive pulmonary disease among managed care patients. Int J Chron Obstruct Pulmon Dis. 2010;5:341-49. doi:10.2147/copd.s13771

26. Kendzerska T, Leung RS, Aaron SD, Ayas N, Sandoz JS, Gershon AS. Cardiovascular outcomes and all-cause mortality in patients with obstructive sleep apnea and chronic obstructive pulmonary disease (overlap syndrome). Ann Am Thorac Soc. 2019;16(1):71-81. doi:10.1513/ AnnalsATS.201802-136OC

27. Schwab P, Dhamane AD, Hopson SD, et al. Impact of comorbid conditions in COPD patients on health care resource utilization and costs in a predominantly Medicare population. Int J Chron Obstruct Pulmon Dis. 2017;12:735-44. doi:10.2147/COPD.S112256

28. Buffel du Vaure C, Dechartres A, Battin C, Ravaud P, Boutron I. Exclusion of patients with concomitant chronic conditions in ongoing randomised controlled trials targeting 10 common chronic conditions and registered at ClinicalTrials.gov: a systematic review of registration details. BMJ Open. 2016;6(9):e012265. doi:10.1136/bmjopen-2016-012265

29. Lloyd JT, Blackwell SA, Wei II, Howell BL, Shrank WH. Validity of a claims-based diagnosis of obesity among Medicare beneficiaries. Eval Health Prof. 2015;38(4):508-17. doi:10.1177/0163278714553661

30. Navickas R, Petric V-K, Feigl AB, Seychell M. Multimorbidity: what do we know? What should we do? J Comorbidity. 2016;6(1):4-11. doi:10.15256/ joc.2016.6.72

31. McPhail SM. Multimorbidity in chronic disease: impact on health care resources and costs. Risk Manag Healthe Policy. 2016;9:143-56. doi:10.2147/ RMHP.S97248

32. Zulman DM, Chee CP, Wagner TH, et al. Multimorbidity and healthcare utilisation among high-cost patients in the US Veterans Affairs Health Care System. BMJ Open. 2015;5(4): e007771. doi:10.1136/bmjopen-2015-007771

33. Wang L, Si L, Cocker F, Palmer AJ, Sanderson K. A systematic review of cost-of-illness studies of multimorbidity. Appl Health Econ Health Policy. 2018;16(1):15-29. doi:10.1007/s40258-017-0346-6 
The Economic Burden of Comorbid Obstructive Sleep Apnea

Among Patients with Chronic Obstructive Pulmonary Disease

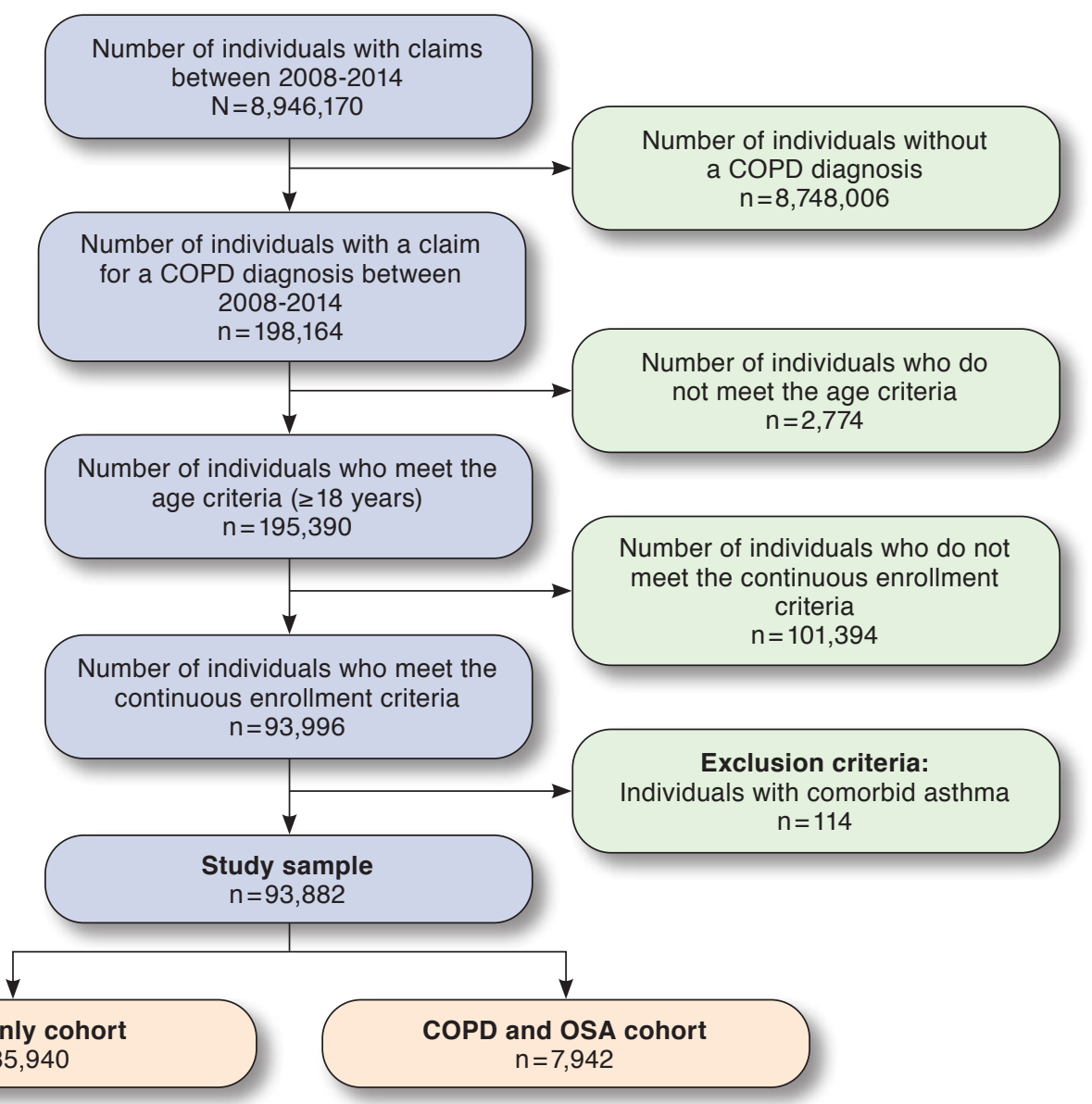

$C O P D=$ chronic obstructive pulmonary disease; $O S A=$ obstructive sleep apnea 
The Economic Burden of Comorbid Obstructive Sleep Apnea

Among Patients with Chronic Obstructive Pulmonary Disease

\begin{tabular}{|c|c|c|}
\hline APPEND & $\begin{array}{l}\text { 12-Month Average } \\
\text { Probability of Having } \\
\text { Hospitalization, ER V } \\
\text { Pharmacy Costs (Part } \\
\text { 2-Part Model) }\end{array}$ & $\begin{array}{l}\text { nal } \\
\text { nd } \\
\text { a }\end{array}$ \\
\hline HCRU Category & $\begin{array}{l}\text { Average Marginal Probability } \\
\qquad(95 \% \mathrm{CI})^{\mathrm{b}}\end{array}$ & $P$ Value \\
\hline Inpatient $^{\mathrm{a}}$ & $0.07 \quad(0.06-0.08)$ & $<0.01$ \\
\hline ER visit ${ }^{\mathrm{a}}$ & $0.05 \quad(0.04-0.06)$ & $<0.01$ \\
\hline Pharmacya & $(0.02-0.04)$ & $<0.01$ \\
\hline $\begin{array}{l}\text { a First part of a 2-p } \\
\text { COPD-OSA cohor } \\
{ }^{b} \mathrm{CI} \text { estimated usin } \\
C I=\text { confidence inte } \\
\text { ER=emergency roc } \\
\text { resource utilization }\end{array}$ & $\begin{array}{l}\text { Lodel: GLM (logit link and binomial } \\
\text { COPD-only cohort (reference). } \\
\text { delta method. } \\
\text { COPD = chronic obstructive pulmor } \\
L M=\text { generalized linear model; HC } \\
A=\text { obstructive sleep apnea. }\end{array}$ & $\begin{array}{l}\text { ution); } \\
\text { ease; } \\
\text { alth care }\end{array}$ \\
\hline
\end{tabular}

